\title{
The application of population based health surveys in pharmacoepidemiologic studies in Norway
}

\author{
Anne Elise Eggen \\ Department of pharmacoepidemiology and pharmacy practice, Institute of pharmacy, University of Tromsø, Norway \\ Correspondence to: Anne Elise Eggen, Institute of pharmacy, University of Tromsø, NO-9037 Tromsø, Norway \\ Telephone: +47 77646343 Telefax: +4777646151 E-mail: elisee@farmasi.uit.no
}

\begin{abstract}
There has been a modest interest in pharmacoepidemiologic research in Norway, especially when considering that drugs are comprising the most common medical treatment and the health care segment with the most rapidly increasing costs in health care. There may be several explanation for the relatively low research output. One may be the generally poor access to information from issued drug prescriptions.

Comprehensive population based health surveys have been carried out in Norway for many years. The health surveys have also been used as a basis for studies of drug use, and they have made it possible to collect important background information associated with drug use. However, health surveys have primarily had their origin in etiological research and the charting of major diseases in the population. Information on drug use has primarily been included as indicators of morbidity. The questions about drug use have therefore been general and covered the use of most drug groups. They have usually included both prescription and non-prescription drugs.

The more recent health surveys include questions on drugs with a more focused approach. More questions are now directed towards more defined health problems and drugs.

Access to individual based drug information from issued prescriptions, with the possibility of doing record linkage studies combining data from the health surveys and prescription information, may contribute significantly to the quality of pharmacoepidemiologic research in Norway.
\end{abstract}

\section{INTRODUCTION}

Pharmacoepidemiologic research requires access to high quality drug use data. Norway has been in the forefront internationally in the development of drug sales statistics. National sales statistics on the use of medications based on wholesale drugs sales to pharmacies since the mid 1970s have been published. The annual publication, Drug consumption in Norway, provide information about the number of dosages sold and the amount of money involved at both national and regional levels ${ }^{1}$. These have been, and still are, very useful, high quality statistics which can be used to evaluate changes in drug sales and to make international comparisons.

Internationally, sales statistics are now more commonly replaced by individual-based prescription registries based on the pharmacies' computer systems. This varies according to whether the pharmacy records are linked to the public social security systems ${ }^{2-5}$, private health insurance associations ${ }^{6,7}$ or primary research enterprises ${ }^{8,9}$.

Generally speaking, prescriptions have rarely been used as a basis for research in Norway because they are classified as confidential information. The lack of prescription registries, and the restricted access to prescription data from the pharmacies, have so far limited the use of prescription information in pharmacoepidemiologic research.

We have, however, for many years had comprehensive population based health surveys in Norway, which have also been used as a basis for studies of drug use. The health surveys makes it possible to collect important background information associated with drug use. One of the information sources for these health surveys is The Central Population Register with personal identity numbers. This register is used to draw random samples or invite defined populations to health surveys. The health surveys include questionnaires providing sociodemographic information, information about lifestyle, symptoms and diseases, and other information which may have relevance for the use of drugs. This may widen both perspectives and objectives of such research. Other national health registers of interest in connection with pharmacoepidemiologic research are The National Mortality Register, The Cancer Registry of Norway and The Medical Birth Registry of Norway. 


\section{CURRENT SOCIAL ISSUES}

The increasing cost of the health service in general and prescription drugs in particular, has been a topic of great debate during the last years. Newer and more expensive drugs replace older, cheaper alternatives. Drugs are now more commonly used to prevent chronic diseases like coronary heart disease and osteoporosis. Larger population groups are now given drugs to prevent possible future diseases.

The use of serum lipid reducing agents illustrate this issue. In 2000, such drugs were sold to a value of 830 million Norwegian kroner (NOK). Sales statistics give an idea of the treatment intensity which corresponds to about $6 \%$ of the population taking a defined daily dose of lipid reducing agents every day of the year ${ }^{1}$. Fig. 1 shows that this consumption is significantly higher than in Sweden and Denmark ${ }^{1,10,11}$.

Treatment with serum lipid reducing agents has a documented effect on patients with familial hypercholesterolemia and in patients with established coronary heart disease. Exactly which patient groups are treated today, and whether they are those who would benefit the most from serum lipid reducing agents is unknown. It is therefore not possible to conclude whether the expenditure is too high or too low based on available drug use data. Work ought to be done to gather more knowledge about those who receive treatment and those who remain untreated. Are we treating those who have been shown to get most benefit from the treatment? Or are there still large groups of untreated patients with severe health problems who should be offered treatment? What is the user pattern like, taking into consideration the users' age and sex, serum lipid levels, blood pressure, diseases and lifestyle factors such as exercise, diet and smoking?

The large increase in the use of serum lipid reducing agents can be attributed to more aggressive treatment of the same condition with higher doses, or to more people with the same indications being treated, or it could imply that new user-groups with new indications receive treatment. An important reason for the increase is probably that serum lipid reducing agents have gained widespread use as a secondary prevention against certain coronary heart diseases ${ }^{12}$. We need pharmacoepidemiologic research in order to enlighten this question of whether the expenditure on lipid reducing treatment is at an acceptable level in Norway; the present debate on this financial aspect is completely inadequate.

Population studies are also necessary to study the long-term effects among different patient groups treated to investigate whether the positive effects on morbidity and mortality shown in controlled clinical studies also may be achieved during everyday clinical practice. This necessitates long-term follow up and access to other health registers. If the answer could be found to any of these questions, more could be known about whether the treatment gives adequate health improvements for the national expense on serum lipid reducing drugs.

\section{PHARMACOEPIDEMIOLOGY STUDIES FROM HEALTH SURVEYS}

Pharmacoepidemiologic research based on health surveys has been scattered and taken place in different research groups. The extent has been modest, especially considering that drugs comprise the most common treatment and one of the most rapidly increasing costs in health care. There may be several explanation for the low research output, one reason may be the poor access to information from issued prescriptions. The health surveys have primarily had their origin in the charting of major diseases in the population and etiological research. Questions about drug use have been included because they may serve as indicators of morbidity. Questions about drug use have therefore been general and covered the use of most drug groups. However, they have included both prescription and non-prescription drugs.

\section{PREVALENCE STUDIES AND USER PATTERNS}

The Norwegian drug sales statistics have been published since the seventies. Great regional differences in drug use were reported, and this prompted projects which sought to explore these differences in order to find possible explanation for the variation ${ }^{13-15}$.

Statistics Norway carried out national health surveys in both 1985 and 1995, where questions of drug use were included. The main purpose of these surveys has been to obtain a general and fairly comprehensive knowledge of health problems in the Norwegian population as a whole and to reveal inequalities regarding health conditions between different groups of the population ${ }^{16,17}$. A representative sample of Norwegian households was invited and interviewed in their own homes by trained interviewers. The questionnaire for children less than 16 years of age should be answered by parents or another adult responsible for the child. The questions on drug use included use during the previous fourteen days.

In a simple study from the Health survey 1985 the proportion of drug users in the population is shown by gender and age. There were 5,454 females and 5,122 males $(0-80+$ years of age) included in the survey (response rate $78.7 \%$ ). Drug use decreased with age in childhood, but the overall age trend showed an increase with age. The gender differences were observed through the childbearing years (15-49 years) and above 70 years of age. Gender differences remained after adjustment for self-reported diseases/health problems. The regional differences in drug use, however, disappeared after adjustment for differences in reported diseases/health problems during the period ${ }^{18}$. 


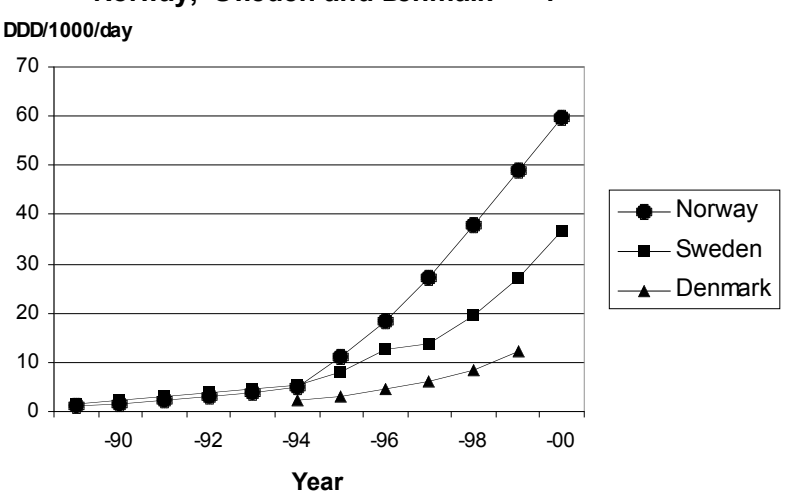

Figure 1. Serum lipid reducing agents. Sales in Norway, Sweden and Denmark ${ }^{1,10,11}$.

The women were also asked about the use of estrogen during menopause, and the changes in estrogen use pattern in the period 1985-95 have been published. The authors showed a marked increase in systemic treatment during the period ${ }^{19}$. The survey in 1995 included almost the same number of participants, and the response rate was $75 \%{ }^{17}$.

National Health Screening Service has carried out systematic health surveys in each county of Norway where information about the use of certain drugs, in particular, use of cardiovascular medication has been recorded. Graff-Iversen has used the health surveys to study cardiovascular risk factors in users of the contraceptive pill and estrogen treatment during menopause $^{20,21}$. See Graff-Iversens article in this issue of the Journal.

In some counties National Health Screening Service has collaborated with universities and other institutions to carry out more extended surveys, such as The Tromsø Health Study, The Finnmark Health Study, The Nord-Trøndelag Health Study (HUNT) and the Hordaland Health Study (HUSK). In these surveys the population has been invited to a health screening including recording of indicators of coronary heart disease, for example body mass index, serum lipids and blood pressure. In addition, the participants filled in questionnaires including topics related to diseases and health problems, use of health services, sociodemography and lifestyle. Subgroups of participants have also been selected for additional follow-up studies. The questionnaires also include several questions regarding drug use. The questions reflect that drug use may be an indicator of different diseases and symptoms, and to distinguish users from non-users. The questions concerning medication cover drug use during the previous fourteen days, month, or week. In the HUNT study they were asked how frequently sleeping pills or sedatives had been used during the past month. In the HUSK study the participants were asked which drugs they had taken the previous day and for which reason. The previous day was selected to minimize possible recall bias (Jørund Straand, University of Oslo, personal communication, 2001). This gives a prevalence of drug use and can be used to describe the proportion of users in the population and to characterize the drug users.

The published studies from these surveys have explored differences in drug use and how health related variables, sociodemographic and lifestyle characteristics may influence drug use.

Bjørndal and Forsén used different health surveys to analyze the huge difference in the psychotropic drug use between men and women, and to find out to what extent the differences could be attributed to differences in self-evaluated psychic health, civil status or social network ${ }^{22}$.

Furu and co-workers have used the Finnmark Health Study from 1987-88 to describe characteristics of drug use $\mathrm{e}^{23,24}$. The study included 17,823 participants aged 20-79 years; the response rate was $84,6 \%$. In their latest paper, the use of non-prescription antacids in the population was studied. Such use could mainly be explained by the occurrence of self-evaluated dyspeptic complaints while lifestyle and sociodemographic factors had little impact ${ }^{24}$.

The different county health surveys carried out by National Health Screening Service have been repeated several times which enable follow-up studies of e.g. long-term effects of blood pressure treatment and mortality. The effectiveness of antihypertensive treatment during everyday clinical practice implies that both selection, compliance and follow-up may vary. The authors concluded that the benefit experienced from the controlled trials turned into an adverse effect of treatment in the population setting, particularly at low pretreatment blood pressure, and when blood pressure increased during treatment ${ }^{25,26}$. These results were heavily debated at the time of publication. A limitation was that the survey did not include information about the type of antihypertensive drug treatment the participants had used.

In some studies, drug prescription data from pharmacies have been linked to information from a population study. This makes it possible to categorize the responders according to the intensity of drug use (frequency and duration of use), and include more detailed information about the type of drugs and the dosages used. Based on the Tromsø Health Study 1986-87 and information from issued prescription from the pharmacies in Tromsø, we analyzed factors which influenced the use of analgesics. In 1986-87 all men and women aged 20-60 and a sample of subjects aged 12-19 living in Tromsø were invited to The Tromsø Health Study. 21,647 (75\%) of the invited attended the screening. Separate studies were undertaken to analyze both self-reported drug use (based on information from the questionnaires regarding use the previous fourteen days $)^{27}$. In one study information regarding sociodemography, lifestyle, symptoms and 
diseases from the Tromsø Health Study were linked to prescription data (one year) for strong analgesics, issued in the community of Tromsø. Combined codeine preparations dominated, and the average amount purchased was 25 defined daily doses/year. The use was mainly sporadic, but regular use did occur. Low selfevaluated health and headache were the most significant predictors. However, daily smoking and low education level were also significant predictors. Drug use increased significantly with age, but the minor gender difference observed became insignificant after adjustment for differences in health problems ${ }^{28}$.

In the Nord-Trøndelag Study (HUNT), the questionnaires were designed to study the use of antidiabetic drugs among type- 1 and type- 2 diabetics. The drug use data were subsequently linked to relevant information from the clinical examination. In the screening survey from 1984-86, all inhabitants in North Trøndelag 20 years or older were invited, and 76,885 (90.3\%) participated in the study. Of the diabetic patients $20 \%$ used insulin, 39\% oral hypoglycemic agents and $41 \%$ did not use any antidiabetic drugs. The mean daily insulin dose per kilo body weight was significantly higher in women than in men. The mean prescribed insulin dose was $14 \%$ higher than the defined daily dose, but the corresponding oral agent dose was lower than the defined daily dose. The authors concluded that sales figures do give a poor estimate of diabetes prevalence in the population due to the high proportion of diabetic patients using no antidiabetic drugs. Corrections are also needed when the defined daily dose system is used to estimate the prevalence of medically treated diabetes ${ }^{29}$.

\section{FURTHER DEVELOPMENTS}

The health surveys usually include multiple objectives and many research questions are included in the same survey. The advantage is the access to a large number of variables. A further development of these studies will be to choose more specific problems and have unambiguous questions at a satisfactory level of detail, with defined end points in order to effectively follow up the treatment. This will result in fewer but betterdefined approaches to drug related problems.

In the questionnaire used during the Oslo Health Study last year, this development towards more explicit approaches is visible. More questions are now directed towards defined health conditions with more detailed questions about the specific drug treatment for the condition (Kari Furu, University of Oslo, personal communication, 2000). There are many drug related issues which are mainly of interest if one can chart the degree of regular or long-term use, and the questions therefore now also focus on the frequency of use. This makes it possible to differentiate between those who take medication regularly, rarely, or not at all.
In the follow-up survey to the Tromsø Health Study in 1994-95, information was acquired about medication used regularly during the preceding week. This was done by sending a short pamphlet in advance to the participants where they were asked to provide the name, strength and dosage of all the medications they had used the previous week. Those who needed help for completing the questionnaire were encouraged to bring their medication with them when attending the survey. This enabled detailed information to be obtained about which pharmacological groups were used in the treatment of each individual. This method is presently being repeated in the Tromsø Health Study 2001, where participants must fill in a questionnaire naming any medication they use regularly and bring the questionnaire with them to the health examination. The forms will be checked during the interview which probably will improve the quality of the information compared with forms completed by the participant alone.

The survey "The Norwegian Woman and Cancer Study" (NOWAC - also called "Women, health and lifestyle"), is a prospective population-based study based on questionnaires sent to a random sample of Norwegian women between the ages of 30 and 70 . The survey has been running since 1991 and up to now, about 105,000 women have responded. The main aim of the survey is to study the effects of using contraceptive pills and estrogen during menopause for the development of cancer. Multiple questions address the use of hormones in addition to information about menstruation, number of children, sociodemography, lifestyle, state of health and different diseases. Along with the questionnaire, the women are provided photos of all branded products of oral contraceptives and hormone replacement preparations that have been available in Norway from 1953 to the present. This may help the respondents to more easily remember which products they have used in their lifetime and for how long they have used the drugs.

The NOWAC survey allow both user-patterns and long-term effects to be studied, and can supply information about user characteristics, current and ever use, type of products, duration of treatment and age at start of treatment. The estrogen studies have also included questions about information sources of the women's knowledge, indications, possible side effects, reasons for stopping treatment and attitudes to estrogen use. A study from the survey on estrogen use during menopause is published by Bakken and Eggen in this issue of the Journal. Studies of possible long-term effects of the use of contraceptive pills and estrogen are in preparation. The NOWAC data will be linked to The Cancer Registry of Norway ${ }^{30}$.

In the future, more of the pharmacoepidemiology studies based on health survey data will probably be longitudinal studies. This is now possible because both The Tromsø Health Study, The Nord-Trøndelag Study, 
the Finnmark Health Study and NOWAC are being repeated in the same population groups including repeated measurements of clinical, biochemical and questionnaire data. This opens up the possibility of studying new drug users separately, and to analyze how treatment changes over time in relation to the course of the health problem.

In health surveys, information on drug use is usually obtained through self-report, with the advantage that information on other relevant conditions can be collected simultaneously. The quality of the information may be greatly improved if access to information from issued prescriptions or a population based drug prescription registry become available. This may also be used for validating the questionnaires. Furthermore, data regarding intensity of treatment and drug use over time may be available as well as detailed information about various drugs used. This may be valuable for follow-up studies on the effects of drugs. For the scientific community, it is important that the proposed National Drug Prescription Registry will allow access to prescription information for the purpose of research. Record linkage studies combining data from the regional health surveys and from the national prescription registry may become a valuable source for future research in the field of pharmacoepidemiology in Norway. This will provide high quality data about drugs as well as clinical and biological measurements such as blood pressure, lipid levels, bone density and glucose levels, and details about sociodemography, lifestyle and diseases obtained from the questionnaires. Access to this combination of population data represent great challenges and possibilities for research and will probably contribute significantly to the further growth of pharmacoepidemiology in Norway.

\section{Summary points}

- There has been a modest interest in pharmacoepidemiology studies in Norway, especially considering that drugs comprise the most common treatment and one of the most rapidly increasing costs in health care.

- In research there has been very restricted access to, or use of, data from issued prescriptions.

- The Norwegian health surveys have focused on diseases and etiology research. Information on drug use has been included primarily as an indicator of morbidity.

- The more recent health surveys include questions on drugs with a more focused approach.

- Better access to individually based drug information from issued prescriptions is needed.

- The possibility of doing record linkage studies including information from prescriptions and health survey data may contribute significantly to the quality of pharmacoepidemiology research in Norway.

\section{REFERENCES}

1. Rønning M, editor. Drug Consumption in Norway 1996-2000. Oslo: WHO Collaborating Centre for Drug Statistics Methodology, 2001.

2. Downey W, Beck P, Stang M, Osei W, Nichol J. Health Databases in Saskatchewan. In: Strom B, editor. Pharmacoepidemiology, Third edn. Chichester: John Wiley \& Sons, 2000: 325-46.

3. Carson J, Ray W, Strom B. Medicaid Databases. In: Strom B, editor. Pharmacoepidemiology, Third edn. Chichester: John Wiley \& Sons, 2000: 307-24.

4. Evans L, MacDonalds T. The Tayside Medicines Monitoring Unit (MEMO). In: Strom B, editor. Pharmacoepidemiology, Third edn. Chichester: John Wiley \& Sons, 2000.

5. Leufkens H, Urquhart J. Automated Pharmacy Record Linkage in The Netherlands. In: Strom B, editor. Pharmacoepidemiology, Third edn. Chichester: John Wiley \& Sons, 2000: 347-60.

6. Shatin D, Drinkard C, Stergachis A. United Health Group. In: Strom B, editor. Pharmacoepidemiology, Third edn. Chichester: John Wiley \& Sons, 2000: 295-306.

7. Saunders K, Davis R, Stergachis A. Group Health Cooperatives of Puget Sound. In: Strom B, editor. Pharmacoepidemiology, Third edn. Chichester: John Wiley \& Sons, 2000: 247-62.

8. Rodriguez L, Peréz-Gutthann S, KJick S. The UK General Practice Research Database. In: Strom B, editor. Pharmacoepidemiology, Third edn. Chichester: John Wiley \& Sons, 2000: 375-86.

9. Gaist D, Sorensen HT, Hallas J. The Danish prescription registries. Dan Med Bull 1997; 44 (4): 445-8.

10. Burman K, editor. Swedish Drug Sales Statistics 1999. Stockholm: Apoteket AB, 2000.

11. Larsen L, editor. Danish Medicinal Products Statistics 1995-99. København: The Danish Medicines Agency, 2000.

12. The Scandinavian Simvastatin Survival Study Group. Randomised trial of cholesterol lowering in 4444 patients with coronary heart disease: The Scandinavian Simvastatin Survival Study (4S). Lancet $1994 ; 344$ (8934): 1383-9. 
13. Haugen H, Hjort P, Waaler H. Drug consumption in the Norwegian counties - large differences, few explanations (In Norwegian). Oslo: The Norwegian Research Council for Science and Humanities. Unit for Health Services Research. National Institute of Public Health, 1978.

14. Bjørndal A, et al. Valuable therapy or social problem? The utilization of psychotropic drugs in Norway (In Norwegian, English summary). Oslo: The Norwegian Research Council for Science and Humanities. Unit for Health Services Research. National Institute of Public Health, 1983.

15. Bjorndal A, Fugelli P. Can regional differences in consumption of tranquillizers and hypnotics be explained by variation in general practitioners' threshold of prescribing? A methodological study. Scand J Prim Health Care 1989; 7 (2): 67-71.

16. Carlson O. Health Survey 1985 Report No: NOS B692. Oslo: Statistics Norway, 1987.

17. Ramm J, Strøm K, Steffensen R. Health Survey 1995 Report No: NOS C516. Oslo: Statistics Norway, 1998.

18. Eggen AE. Patterns of medicine use in a general population (0-80 years). The influence of age, gender, diseases and place of residence on drug use in Norway. Pharmacoepidemiol Drug Saf 1997; 6 (3): 179-87.

19. Graff-Iversen S, Ramm J. Østrogenbruken øker [Increasing use of estrogens]. Samfunnsspeilet 1997 (2): 55-8.

20. Graff-Iversen S, Tverdal A, Stensvold I. Cardiovascular risk factors in Norwegian women using oral contraceptives: Results from a cardiovascular health screening 1985-88. Contraception 1996; 53 (6): 337-44.

21. Graff-Iversen S, Stensvold I, Lund-Larsen PG, Orozco-Nodarse L, Tverdal A, Urdal P. Serum lipids in postmenopausal or perimenopausal women using estrogen alone, estrogen with levonorgestrel, or estrogen with norethisterone, compared with nonusers: Results from a crossectional study in two Norwegian counties 19851988. J Clin Epidemiol 1998; 51 (12): 1311-6.

22. Bjørndal A, Forsén L. A study of psychotropic drug use with data from the Nord-Trøndelag Health Survey 1984-86 (In Norwegian, English summary). Oslo: The Norwegian Research Council for Science and Humanities. Unit for Health Services Research. National Institute of Public Health, 1989.

23. Furu K, Straume B, Thelle D. Legal drug use in a general population: Association with gender, morbidity, health care utilization, and lifestyle characteristics. J Clin Epidemiol 1997; 50 (3): 341-9.

24. Furu K, Straume B. Use of antacids in a general population. The impact of health-related variables, lifestyle and sociodemographic characteristics. J Clin Epidemiol 1999; 52 (6): 509-16.

25. Thurmer HL, Lund-Larsen PG, Tverdal A. Is blood pressure treatment as effective in a population setting as in controlled trials? Results from a prospective study. J Hypertens 1994; 12 (4): 481-90.

26. Thelle DS, Thurmer H, Lund-Larsen PG, Tverdal A. Is blood pressure treatment as effective in a population setting as in controlled trials? Results from a prospective study. $J$ Hypertens 1995; 13 (5): 567-9.

27. Eggen AE. The Tromso study: Frequency and predicting factors of analgesic drug use in a free-living population (12-56 years). J Clin Epidemiol 1993; 46 (11): 1297-304.

28. Eggen AE. The use of controlled analgesics in a general population (15-59 years) - The influence of age, gender, morbidity, lifestyle and sociodemographic factors. Pharmacoepidemiol Drug Saf 1996; 5 (2): 101-11.

29. Midthjell K, Holmen J, Bjorndal A. Types of diabetes treatment in a total, Norwegian, adult population. The Nord-Trondelag diabetes study. J Intern Med 1994; 236 (3): 255-61.

30. Kumle M, Lund E, Weiderpass E, Braaten T. Oral contraceptive use as a risk factor for breast cancer, "The Women's Lifestyle and Health Study". Nor J Epidemiol 2000; 10 (Suppl. 2): 18. 